\title{
El arte de la educación en México
}

\section{The art of education in México}

\author{
DIAZ-LEDEZMA, José De La Cruz†** \\ Instituto Educativo Dolores del Río, extensión Santiago Papasquiaro, Dgo.
}

ID $1^{\mathrm{er}}$ Autor: José De La Cruz, Diaz-Ledezma / ORC ID: 0000-0002-9528-9235, CVU CONACYT ID: 852582

DOI: $10.35429 / J T M S .2019 .16 .5 .45 .52$

Recibido 11 de Junio 2019; Aceptado 30 Septiembre, 2019

\section{Resumen}

El presente artículo presenta una visión de la relación que se puede establecer entre educación y arte en nuestro país, partiendo del análisis de los objetivos de la formación docente y el papel que este juega en el proceso educativo, presenta un análisis crítico de las intencionalidades de la educación en el nivel básico y la formación de docentes. Es un estudio intencional de la función del docente en un país donde el papel del Estado educador asume las funciones de diseñar, orientar, organizar y dirigir, a través de la escuela los destinos de las nuevas generaciones, formándolas en un proceso educativo de acuerdo con los intereses y necesidades de sí mismo. Objetivo: identificar la influencia que el Estado ejerce a través de la educación, en los docentes en activo, en la formación de los docentes y en los alumnos de los diferentes niveles educativos de nuestro país. Metodología: investigación documental cualitativa, donde se analizan diferentes momentos de la formación de los docentes a la luz de la teoría, leyes educativas y demás relacionados con el tema. Contribución: originar puntos de discusión en torno al proceso educativo y los fines del Estado en materia educativa, desde la formación de los docentes y su desempeño en el aula como agente no de transformación, sino como agente reproductor de los deseos del Estado.

Educación, Formación Docente, Fines

\begin{abstract}
This article presents a vision of the relationship that can be established between education and art in our country, starting from the analysis of the objectives of teacher education and the role it plays in the educational process, presents a critical analysis of the intentionality of Basic education and teacher training. It is an intentional study of the role of the teacher in a country where the role of the educating State takes on the functions of designing, orienting, organizing and directing, through the school the destinies of the new generations, forming them in an educational process according to the interests and needs of. Objective: to identify the influence that the State exerts through education, in active teachers, in the training of teachers and in students of the different educational levels of our country. Methodology: qualitative documentary research, where different moments of teacher training are analyzed in the light of theory, educational laws and others related to the subject. Contribution: originate discussion points around the educational process and the State's aims in educational matters, from the training of teachers and their performance in the classroom as an agent not of transformation, but as a reproductive agent of the wishes of the State.
\end{abstract}

Education, Teacher Training, Purposes

Citación: DIAZ-LEDEZMA, José de la Cruz. El arte de la educación en México. Revista Transdisciplinaria de Estudios Migratorios. 2019, 5-16: 45-52

\footnotetext{
*Correspondencia al Autor (correo electrónico: jdelacruzdiazl@ gmail.com)

$\dagger$ Investigador contribuyendo como primer autor.
} 


\section{Introducción}

El artículo que aquí presentó se orienta hacia el análisis crítico de la función docente en un Estado educador que determina las orientaciones y los fines de la formación no sólo de los docentes sino de la población. En un primer momento se plantea la idea de la preparación y el desmepeño y alguna preguntas orientadoras del análisis, donde cada una de las decisiones que toma tienen cierta orientación política, económica o social.

Posteriormente se realiza un desarrollo temático que abunda sobre la fundamentación teórica, la metodología empleada, algunas conceptualizaciones de arte y educación, el papel del Estado en la Educación. Realizado lo anterior se hace un acercamiento analítico de el arte en la educación con la finalidad de establecer en que momento la educación puede considerarse un arte y cual es su procedencia, en tal sentido se plantea también una crítica en relación con la el tema.

Finalmente se plantea una discusión teórica y las conclusiones que se derivan de la realización de este trabajo.

La importancia del tema radica proincipalmente en maracar una linea que permita identificar el grado de independencia que posee el docente al desarrollar su labor educativa y al plantear el trabajo aúlico en diferentes momentos.

La parte que es de llamar la atención es la aplicación de una investigación de corte documental cualitativo que nos permite explorar el tema como un acercamiento a la realidad que vive la educación en nuestro país

\section{Fundamentos Teóricos}

Para la realización del presente se tomaron en cuenta aportaciones de diversos autores que han escrito y desarrollado teoría en torno a la educación, entre ellos, encontramos a Pierre Bourdieau con su teoría de la reproducción y la violencia simbolica, a Emile Durkheim con su trabajo sobre educación y sociología, a Jacques Delors con la Educación encierra un tesoro, además del Plan y Programas de Estudio emitidos por la Secretaría de Educación Pública, así como algunas leyes que regulan el trabajo docente.
De cada uno de ellos se tomó lo necesario para dar coherencia a este trabajo, considerando que no es un trabajo exhaustivo, por lo que la exploración del tema en cuestión queda abierto a una mayor investigación, reflexión e interpretación.

\section{Metodología}

El presente artículo se enmarca en un enfoque cualitativo de investigación documental exploratoria y descriptiva, con una orientación hermenéutica, es decir desde una orientación donde se funden los aportes teóricos y las interpretaciones del autor. Cualitativo porque pretende a la luz de la interpretación y el análisis de la teoría encontrar los elementos que permitan orientar el punto de vista sobre el tema de estudio, como lo dice Sampieri (2016) "la investigación cualitativa se enfoca en comprender los fenómenos, explorándolos desde la perspectiva de los participantes en un ambiente natural y en relación con su contexto", en este caso la investigación desarrollada busca comprender el fenómeno de la formación docente en México, los alcances de la participación del Estado en la educación y la participación de los docentes en la misma.

Es exploratoria porque la revisión de la literatura nos lleva únicamente al conocimiento inicial del fenómeno, interpretativa porque en este caso se analizan e interpretan diversas posiciones teóricas y se comparan con las posiciones del Estado como el gran promotor de un sistema educativa que define las orientaciones de la educación en el país. Es descriptivo porque se plantean dentro de la misma reflexión las experiencias que, como docente, realiza el autor. El objetivo de este estudio es identificar la participación del Estado la formación docente.

\section{Arte. Un acercamiento a su definición}

Las preguntas planteadas en el párrafo anterior conducen el abordaje de los dos términos iniciales: arte y educación. Para lograrlo es necesario partir de alguna definición concreta que nos indique el significado de éstas, por ello debemos ir hacia el pasado y hacer una mirada retrospectiva que nos permita conocer el origen, por ello es que se recurre directamente a la teoría, haciendo una búsqueda documental, que nos permita realizar una interpretación del tema en cuestión. 
Realizando una indagación documental que nos ayude, podemos encontrar los antecedentes etimológicos de arte, en griego como $\tau \varepsilon ́ \chi v \eta$ (tekné) y en latín como "ars" "artis", y que puede significar en ambos casos "arte", sin embargo, el vocablo no hace referencia a lo que actualmente conocemos como arte, más bien se refiere a la técnica con la que se realizaba alguna actividad.

Ferrater (1965) en su diccionario de filosofía plantea que se puede hablar de distintos tipos de arte, por ejemplo, el arte de pensar, de vivir, de actuar, entonces '"arte' significa en este sentido una cierta virtud o habilidad para hacer o producir algo" (pág. 142). En esta dirección es posible identificar que, según Sonia Vicente,

"...en el concepto griego de técnica, como en la noción posterior (latina y medieval) de arte, se encierran diversos significados: por un lado, ambos implican un saber hacer, y suponen que ese saber es conocimiento de las reglas que rigen el hacer.

Para los antiguos y los medievales técnica y arte significaban destreza, es decir, la destreza que se requería para construir un objeto." (2003, pág. $s / p$ )

Entonces arte está relacionado directamente con el hacer, con un modo de proceder en la elaboración de un producto o la realización de una actividad, arte es pues el proceso que se desarrolla cuando algo es llevado a término considerando características especiales que lo hacen único o diferente.

La elaboración de algún producto o la realización de una actividad (realizar algo) cualquiera que esta sea, requieren de tener un conocimiento que les permita hacerlo de manera adecuada, dicho conocimiento procede de una formación previa, de una formación adquirida a través de un proceso educativo, donde el aprendizaje y la enseñanza forman un dúo inseparable, y este dúo es representado por el aprendiz y el instructor. Es necesario pues haber pasado por una instrucción, un adiestramiento o una educación en el sentido de proceso de enseñanza y aprendizaje.

\section{Educación}

En tal caso, la educación puede ser definida o considerada como el resultado de un proceso de enseñanza y aprendizaje que involucra una relación de sujeción a las normas del maestro o el mentor, aunque también puede ser considerada como actitudes o valores frente a situaciones que implican una buena conducta ante la sociedad, así se oye decir "no tiene educación" cuando alguna persona pasa y no ofrece el saludo a otros; también se escucha decir "de que le sirve la educación que tiene si no es capaz de pedir adecuadamente las cosas", en este aspecto se refiere a decir "por favor" antes o después de una solicitud oral, podemos observar en ambos casos que la educación es concebida como un buen trato a los demás o como una forma de solicitar algo.

La educación es un acto humano que permite transmitir a las nuevas generaciones los valores, actitudes, conocimientos y el bagaje cultural de una sociedad en constante transformación que irremediablemente se perpetúa a través de ella.

Etimológicamente el término educación procede del latín "...proviene de dos vocablos, ...Estos vocablos son los siguientes: "educare", que traducido al idioma castellano significa: criar, nutrir, alimentar; "ex-ducere", que en nuestra lengua quiere decir extraer, sacar-de, llevar", esencialmente, educar también tiene una relación con el hacer, en este caso es el hacer docente, lo que el maestro realiza; Durkheim (1975) por su lado dice que la educación no es, para lo sociedad, más que el medio con que prepara en el espíritu de los niños las condiciones esenciales de su propia existencia, y sentencia:

La educación es la acción ejercida por las generaciones adultas sobre las que todavía no están maduras para la vida social. Tiene por objeto suscitar y desarrollar en el niño cierto número de estados físicos, intelectuales y morales, que exigen de él la sociedad política en su conjunto y el medio especial al que está particularmente destinado. (Durkheim, 1975, pág. 53) 
La educación en esa doble acepción implica acción, movimiento, y todo movimiento trae consigo hacer algo, pero el hacer en la educación está orientado, acotado, condicionado y generalmente regulado por la misma formación que el Estado hace de los maestros.

\section{El Estado en la educación}

Esta regulación es identificable en los objetivos planteados en los planes de educación normal o de formación de docentes, sean estos en instituciones públicas o privadas, pues en estos planes, como en los de educación básica, se encuentra la mano del estado educador.

Cuando el Estado se convierte en el rector de la educación casi siempre lo hace con una doble intención y ésta puede ser entendida como un proceso educativo, a la vez que de adoctrinamiento; educativo porque impulsa desde sus adentros la formación del pueblo y de adoctrinamiento porque indica desde la misma elaboración de planes y programas la dirección que ha de tomar el país. Así cada momento de la historia de nuestro país ha reflejado las intenciones del Estado para poder mantener el estado de cosas tal como están o con una visión de futuro que obliga a los docentes a ser partícipes de esa doble intención.

La vida educativa de nuestro país ha estado marcada por ordenanzas educativas que parten del centro y han de ser traducidas por los docentes en todo momento; por ejemplo, después de la Guerra de Reforma, cuando los liberales logran hacerse del poder político buscan una orientación educativa que les permita dar a conocer sus intenciones y formar a la población para poder darle cabida al laicismo, que en aquel periodo se definía como la separación del Estado y la Iglesia, dando a cada uno su ámbito de competencia: el Estado en el ámbito civil y la Iglesia en el ámbito espiritual.

Por ello era necesario encontrar una orientación filosófica que permitiera implantar el laicismo como orientación política del grupo en el poder. El positivismo, traído por Barreda a nuestro país, fue la orientación que permeó en el ámbito educativo durante las tres últimas décadas del siglo XIX.
Con el positivismo se transformaron planes y programas, se dio una orientación científica a la educación, se crearon nuevas instituciones que respondieran a las necesidades del país en ese momento específico de la historia nacional, en otras palabras se introdujo una nueva forma de pensar y como consecuencia de ello, también se preparaba a la población para vivir en esa nueva manera de concebir la vida nacional. En este caso la acción pedagógica hubo de transformarse para lograr establecer lo que el Estado creía era lo adecuado para la población.

Las ordenanzas educativas no representan otra cosa más que la capacidad del Estado de ejercer el poder sobre el resto de las instituciones, sean éstas públicas o privadas, y ese ejercicio es impuesto a los demás por diferentes medios, entre ellos la educación; es, como lo dice Bourdieu (1981), una violencia simbólica porque se imponen significaciones haciéndolas creer legítimas, necesarias y obligatorias para toda la población, así toda acción pedagógica se orienta hacia la reproducción de lo que el Estado desea.

De esta manera, la acción pedagógica, es decir, la acción del docente, forma parte de la reproducción y se convierte a su vez, como lo mencioné anteriormente, en una violencia simbólica que responde a los intereses del Estado, porque como lo expresa Bourdieu "Toda acción pedagógica (AP) es objetivamente una violencia simbólica en tanto que imposición, por un poder arbitrario, de una arbitrariedad cultural" (1981, pág. 45). Y esas regulaciones que he mencionado en los párrafos anteriores se viven y son objetivadas por los docentes en su ámbito de acción: la escuela.

Entonces la escuela se convierte en ese gran laboratorio que permite la reproducción de esquemas culturales de una sociedad y un Estado que responde a las necesidades de ¿quién? ¿la población? ¿la clase política? ¿la clase económica? ¿la clase dominante? dependiendo de quien lea el artículo dará respuesta a la pregunta planteada. 


\section{Formación docente. Intencionalidades}

Volviendo al tema inicial, (¿es la educación un arte o el arte procede del docente?) podemos encontrar que el docente ha sido formado de acuerdo a las características que el Estado ha designado y que en determinado momento le son necesarias, sin embargo, pese a esa modelación, el docente debe realizar su quehacer de la mejor manera, debe ser capaz de inculcar y llevar a los estudiantes a un estado de aprendizaje que les permita acceder a nuevos conocimientos, pero lo que el docente no debe olvidar es que debe cumplir con las regulaciones y reglamentos que el mismo Estado ha diseñado para su acción, como lo dice José Ángel Pescador Osuna "al maestro le compete el papel de agente multiplicador o reproductor, no sólo de conocimientos, sino también, y quizá lo más importante, de ideología predominante en la cual se ubica" (Pescador, 1983)

Como muestra de esas regulaciones, es posible identificar en los objetivos del plan de estudios de Normal Primaria de 1975; los siguientes:

"2. Mantengan un criterio profesional "por completo ajeno a cualquier doctrina religiosa" y, basados en los resultados del progreso científico, estén dispuestos a luchar contra la ignorancia y sus efectos, las servidumbres, los fanatismos y los prejuicios.

3. Observen una actitud democrática y consideren "a la democracia no solamente como una estructura jurídica y un régimen político, sino como un sistema de vida fundado en el constante mejoramiento económico, social y cultural del pueblo".

8. Sustenten "los ideales de fraternidad e igualdad de derechos de todos los hombres"; sin distinción de razas, sectas, grupos, sexos, individuos, condición social o económica.

17. Sean promotores del cambio social, con un criterio revolucionario fundado en nuestro pasado histórico." (Diario Oficial de la Federación, 1975).
Cada uno de los objetivos extraídos del plan de estudios de educación Normal y que fueran publicado en el citado diario el 26 de agosto de 1975, plantea por sí mismo la orientación que debía tener la formación de Profesores de Educación Primaria, y como consecuencia la formación de los alumnos del mismo nivel.

Obsérvese que los objetivos marcan la orientación de la formación docente y su posterior aplicación en el aula, logrando crear maestros que estén ajenos a cualquier doctrina religiosa y basen sus enseñanzas en el crecimiento científico para acabar con prejuicios y fanatismos, sin embargo, lo anterior antoja una lucha titánica debido a la religiosidad que el pueblo mexicano tiene y el sentimiento de amor a la virgen de Guadalupe; se trata, además, crear maestros que orienten los procesos educativos relacionados con la democracia, en un país, donde la misma se encuentra ajena a la toma de decisiones, pues estas son tomadas en las altas esferas de la política y la economía de nuestro país.

Los siguientes dos son tan idealistas como los primeros, pues en verdad se puede asegurar que el maestro, docente, profesor, llámesele como se le llame, nunca tuvo -ni tieneen sus manos la oportunidad de ser un agente de cambio social con espíritu revolucionario, porque ese espíritu le pertenece a las instituciones emanadas de la revolución de inicios del siglo pasado; además, como podía hablar de fraternidad si se enfrentaba a situaciones de cacicazgo en diferentes partes del país, donde igual que ahora, los "bien nacidos" son merecedores de "todo" mientras los otros deben subsistir con los resultados emanados de su esfuerzo diario.

En cierto modo la función que el docente debía desempeñar se enmarca en lo siguiente: no hablar de religión, poner sus ojos en la ciencia y sus avances $y$ transmitirlo a las nuevas generaciones, fundar las acciones de la sociedad en una democracia fingida que sólo beneficia a los poderosos, ser un agente de cambio que impulsara el reconocimiento a los que por la revolución nos legaron el país que hoy tenemos. 
Para la década de los noventa, en el marco del federalismo educativo, y en las reformas educativas que se dieron en el Acuerdo Nacional para la Modernización de la Educación Básica (ANMEB) la función del docente cambia, como también cambian los objetivos de la formación docente.

En esta nueva formación se pretendía que el docente se convirtiera en investigador y a la luz de las teorías cognitivas llevara a los niños a ser constructores de su propio aprendizaje, así el docente toma el rol de propiciador antes que reproductor del conocimiento, es decir, el docente se convierte en el mediador entre el conocimiento y el alumno, dejando a un lado el papel que le asignaba Pescador Osuna (véase pag. 7).

Sin embargo, con el ANMEB se instituyó, como atribución federal, la facultad de regular un sistema nacional de formación, actualización, capacitación y superación profesional para maestros de educación básica a fin de mejorar la calidad profesional de éstos.

Lo anterior nos lleva a inferir que aún los docentes estaban sujetos a ese espacio de la reproducción. Aun y cuando se les daba cierta autonomía para trabajar, también se les marcaba el rumbo de lo que debía enseñar y como enseñarlo. Es decir, continuaba siendo, en cierto modo, un reproductor de lo que el Estado definía, como podemos advertirlo en el siguiente párrafo:

"Se reiteró el papel del Estado como promotor de la educación media superior y superior, de tipos y modalidades educativas alternas, como la educación de adultos. Asimismo, se sentaron las bases de un esquema de formación para el trabajo pertinente $y$ vinculado con las necesidades del sector productivo" (El desarrollo de la educación. Informe Nacional México, 2001)

Hasta antes de este párrafo se ha hecho referencia al hacer docente en educación básica. Lo que la cita del párrafo anterior nos indica es que en los niveles posteriores a la educación básica también la acción docente se presenta como una reproducción de las necesidades del Estado, traducidas en una plan o programa de estudios en cuyo caso la promoción recae en el Estado mismo.
Pero con la salvedad de que algunas instituciones de nivel superior poseen autonomía en los contenidos educativos que se deben desarrollar, sin dejar de lado las necesidades del país; sin embargo, la formación docente y la elaboración de plan y programas de estudios de educación normal es responsabilidad única del Estado y sus órganos de planeación educativa a través del Ejecutivo Federal y de la Secretaría de Educación Pública (Art $3^{\circ}$ Constitucional).

De tal modo que los nuevos docentes llevan en su formación profesional las bases sociopolíticas que han de ser traducidas a los alumnos de nivel básico y esto, por donde quiera que se vea, es el principio de un adoctrinamiento ideológico del Estado, con la finalidad de tener y mantener el poder político, social, cultural y económico sobre la población.

Así pues, la educación recae directamente en el Estado, independientemente de la autonomía de algunas instituciones y debe responder a las necesidades actuales de la nación y el gobierno en turno. Desde este punto es indudable que definir el término educación es de suyo complejo, pues en él inciden diferentes posiciones ideológicas que le dan la orientación que en determinado momento y lugar se hacen necesarios.

\section{El arte de la educación}

Si como lo mencioné en las preguntas iniciales el arte en la educación procede del docente, entonces es función principal del docente la de traducir los deseos de un Estado que día a día orienta el desarrollo basado en la educación, y si el arte procede del docente es porque él debe ser capaz de hacer su trabajo de la mejor manera, mostrando, enseñando, innovando, buscando soluciones a problemas que en esencia no le competen.

Esos problemas a los que hago referencia pueden ser, entre otros, que los alumnos tengan problemas en casa o que no se alimenten adecuadamente y éstos, indudablemente, se traducen en un problema de aprendizaje que el docente debe solucionar utilizando recursos, medios y materiales didácticos y emocionales que ayuden al alumno a tener un mejor aprovechamiento en la escuela. 
Si por otro lado, el arte está dentro de la educación como actividad, entonces los docentes deben estar preparados para desarrollar ese arte de la mejor manera y que mejor preparación, que el trabajo diario y la profesionalización constante de su tarea, pero sin olvidar que el docente sólo desarrolla lo que el Estado ha diseñado para ser reproducido en el aula, convirtiendo entonces al docente como un mero ejecutor de plan y programas, sin oportunidad de transformar la realidad que viven diariamente los alumnos y la población.

$\mathrm{Si}$ consideramos que el arte y la educación son dos actividades del todo humanas y sociales, entonces podemos asociarlas en el hecho de la actividad misma, pero sin poner uno por encima de la otra o viceversa, lo que sí no debemos olvidar es que el arte de la educación es un juego bien planeado por el Estado, que confiere a los docentes la tarea de adoctrinamiento y de ejercer una violencia simbólica al ser reproductor de los deseos del mismo.

La educación de nuestro país ha respondido a las necesidades de los grupos políticos y económicos, que en aras de un mejor desarrollo han implementado reformas educativas de las cuales la participación del docente se limita únicamente a la reproducción, y es en este espacio donde el arte de la educación en México solo ha servido para formar las personas que son necesarias.

En este contexto de formación docente, se responde también a las necesidades planteadas a escala mundial, donde se delinean las tareas que debe asumir el docente, desde la UNESCO, con las propuestas planteadas por Delors (1996) en La educación encierra un tesoro, los nacionalismos obtusos deberán dejar paso al universalismo, los prejuicios étnicos y culturales a la tolerancia, a la comprensión y al pluralismo, el totalitarismo a la democracia en sus diversas manifestaciones, y un mundo dividido -en que la alta tecnología es privilegio de unos pocos- a un mundo tecnológicamente unido.
Lo anterior trae consigo un nuevo docente, un docente que sea, en cierto modo el pilar del desarrollo del país, a quien se le exige cumplir con su cometido: la formación de las nuevas generaciones en y para el desarrollo, sin embargo, esta exigencia va acompañada de ciertas condiciones que debe asumir el Estado: "hay que empezar por mejorar la contratación, la formación, la situación social y las condiciones de trabajo del personal docente" (Delors, 1996, pág. 155), de esta manera, el Estado mexicano, a partir de las reformas realizadas a la Constitución Política y la publicación de la Ley del Servicio Profesional Docente(2013), se reposiciona como el único que puede contratar el personal docente necesario para la misión de educar.

La contratación se realizará de acuerdo a los resultados de un examen en el que participan egresados de las Escuelas Normales y demás instituciones formadoras de docentes de Educación Básica del país públicas y particulares con autorización; a los egresados de las Instituciones de educación Superior del país, públicas y particulares, con reconocimiento de validez oficial, formadoras de los profesionales que se requieren para los diferentes tipos de evaluación por: nivel educativo, tipo de servicio, modalidad, asignatura, tecnología o taller, y lo anterior es constatable en las convocatorias estatales y nacionales de la SEP para el ingreso al servicio profesional docente.

Surge aquí una nueva pregunta ¿Garantiza el obtener una calificación "idóneo" en un examen resultados iguales en el proceso de enseñanza aprendizaje?

La pregunta anterior queda abierta para quienes, en los altos puestos de dirección del sistema educativo nacional, se encargan de diseñar toda la ingeniería educativa del país, para que mediante análisis minuciosos y con los documentos con los cuales cuentan den respuesta a la misma, si la respuesta es negativa, entonces vuelvan hacia las escuelas formadoras de docentes y dótenlas de los insumos necesarios que permitan una formación docente que responda a las necesidades del país y no de la política económica en turno. 


\section{Discusión}

Los autores que se han rescatado en este artículo y que definen la participación del Estado como agente que puede llegar a determinar los objetivos en la educación y ejercer el predominio en la misma son, por un lado, José Ángel Pescador Osuna, quien en su momento fuera secretario de educación pública en México y Pierre Bourdieu, ambos coinciden en que el papel del Estado en la educación es tal que de manera tácita o explícita determina la acción pedagógica hacia los linderos que desea, obligando de esta manera a que el docente actúe como se le indica y no de manera natural, lo que en determinado momento convierte al docente en mero reproductor de las intencionalidades del sistema. Por otro lado, Delors plantea que, para que haya una educación con la calidad que se desea, el Estado deberá realizar algunos cambios de fondo en el sistema educativo relacionados con la función docente, estos cambios hacen alusión a la formación, la situación social y las condiciones de trabajo de los docentes.

Sin embargo, es notorio, como se observa en los medios de comunicación masiva, que el culpable (o los culpables) de la baja calidad de la educación es el docente, pero no puedes culpar a alguien de los resultados de las acciones que tú mismo estas proponiendo se realicen.

\section{A modo de conclusión}

El arte de la educación en México, y tal vez en el mundo, exhibe todos aquellos intereses de grupos, donde la escuela juega el principal papel, al hacer de la acción docente y de la escuela misma un vehículo para implantar los deseos del Estado.

El arte de la educación en nuestro país ha de ser entendida como la orientación, reglamentaciones, formación docente $y$ elaboración e implantación curricular como un medio para lograr el tipo de ciudadano que el mismo Estado desea.

El arte de la educación se aleja mucho de la actividad creadora, que en todo caso es lo que llamaríamos arte, para convertirse en una actividad reproductora, donde el papel principal lo juegan los maestros y los alumnos, en un espacio dedicado para ello: la escuela.
La actividad creadora queda de lado cuando el docente debe traducir el currículum y hacerlo asequible a los alumnos, sin darse cuenta plena que solo está contribuyendo a esa violencia simbólica definida por Bourdieu.

\section{Referencias}

Bourdieu, P. J. (1981). La reproducción. En La reproducción. Elementos para una teoría de la enseñanza (págs. 44-106). Barcelona, España: Laia/Barcelona.

Delors, J. (1996). La educación encierra un tesoro. Madrid, España: Grupo Santillana de Ediciones.

Diario Oficial de la Federación. (26 de Agosto de 1975). Recuperado el 2 de Mayo de 2017, de http://www.dof.gob.mx/index.php:

http://www.dof.gob.mx/nota_detalle.php?codig $\mathrm{o}=4814146 \&$ fecha $=08 / 09 / 1975$

Durkheim, É. (1975). Educación y sociología (1 ${ }^{\mathrm{a}}$ ed.). (J. M. Liarás, Trad.) Barcelona, España: Península.

(2001). El desarrollo de la educación. Informe Nacional México. Secretaría de Educación Pública, Dirección General de Relaciones Internacionales. México: SEP. Obtenido de $\mathrm{http} / / /$ bibliotecadigital.conevyt.org.mx/coleccio nes/documentos/desarrolloeducativo.pdf

Ferrater, M. J. (1965). Diccionario de filosofía ( $5^{a}$ edición ed., Vol. 1). Buenos Aires, Argentina: Sudamricana.

Pescador, O. J. (Octubre-Diciembre de 1983). Revista Perfiles Educativos. (UNAM, Ed.) Recuperado el 2 de Mayo de 2017, de http://www.iisue.unam.mx/perfiles/:

$\mathrm{http} / / / \mathrm{www}$.iisue.unam.mx/perfiles/busqueda.ph $\mathrm{p}$ ?busqueda $=\mathrm{La}+$ formacion $+\mathrm{del}+$ magisterio $+\mathrm{en}$ + Mexico

Sánchez, d. S. (2010). Arte y educación. Diálogos y antagonismos. Recuperado el 25 de abril de 2017, de http://diposit.ub.edu/dspace/: http://diposit.ub.edu/dspace/handle/2445/59348

Vicente, S. (2003). Arte y ciencia. Reflexiones en torno a sus relaciones. Huellas, 85-94. 